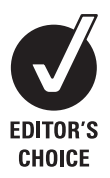

See Editorial, p 1553

${ }^{1}$ The Hatter Cardiovascular Institute, University College London Hospital, London, UK;

${ }^{2}$ The Heart Hospital, University College London Hospitals NHS

Trust, London, UK

\section{Correspondence to:}

Professor D M Yellon, The Hatter

Cardiovascular Institute,

University College London

Hospital, 67 Chenies Mews,

London WC1E 6HX, UK;

d.yellon@ucl.ac.uk

Accepted 23 September 2008

Published Online First

8 June 2009

\title{
Remote ischaemic preconditioning reduces myocardial injury in patients undergoing cardiac surgery with cold-blood cardioplegia: a randomised controlled trial
}

\author{
V Venugopal, ${ }^{1}$ D J Hausenloy, ${ }^{1}$ A Ludman, ${ }^{1}$ C Di Salvo, ${ }^{2}$ S Kolvekar, ${ }^{2}$ J Yap, ${ }^{2}$ \\ D Lawrence, ${ }^{2} \mathrm{~J}$ Bognolo, ${ }^{2}$ D M Yellon ${ }^{1}$
}

\begin{abstract}
Background: Remote ischaemic preconditioning (RIPC) induced by brief ischaemia and reperfusion of the arm reduces myocardial injury in coronary artery bypass (CABG) surgery patients receiving predominantly crossclamp fibrillation for myocardial protection. However, coldblood cardioplegia is the more commonly used method world wide.
\end{abstract}

Objective: To assess whether RIPC is cardioprotective in CABG patients receiving cold-blood cardioplegia.

Design: Single-centre, single-blinded, randomised controlled trial.

Setting: Tertiary referral hospital in London.

Patients: Adults patients (18-80 years) undergoing elective CABG surgery with or without concomitant aortic valve surgery with cold-blood cardioplegia. Patients with diabetes, renal failure (serum creatinine $>130 \mathrm{mmol} / \mathrm{l}$ ), hepatic or pulmonary disease, unstable angina or myocardial infarction within the past 4 weeks were excluded.

Interventions: Patients were randomised to receive either RIPC ( $n=23)$ or control $(n=22)$ after anaesthesia. RIPC comprised three 5 min cycles of right forearm ischaemia, induced by inflating a blood pressure cuff on the upper arm to $200 \mathrm{~mm} \mathrm{Hg}$, with an intervening $5 \mathrm{~min}$ reperfusion. The control group had a deflated cuff placed on the upper arm for 30 min.

Main outcome measures: Serum troponin T was measured preoperatively and at 6, 12, 24, 48 and $72 \mathrm{~h}$ after surgery and the area under the curve (AUC at $72 \mathrm{~h}$ ) calculated.

Results: RIPC reduced absolute serum troponin T release by $42.4 \%$ (mean (SD) AUC at $72 \mathrm{~h}: 31.53$ (24.04) $\mu \mathrm{g} /$

I.72 h in controls vs 18.16 (6.67) $\mu \mathrm{g} / \mathrm{l} .72 \mathrm{~h}$ in RIPC; $95 \%$ Cl 2.4 to 24.3; $p=0.019$ ).

Conclusions: Remote ischaemic preconditioning induced by brief ischaemia and reperfusion of the arm reduces myocardial injury in CABG surgery patients undergoing cold-blood cardioplegia, making this non-invasive cardioprotective technique widely applicable clinically.

Trial registration number: NCT00397163.

Coronary artery disease is the leading cause of death globally, and coronary artery bypass graft (CABG) surgery is one of the established procedures for the treatment of patients with severe coronary artery disease. The risk profile of patients who are referred for cardiac surgery continues to change with factors such as the ageing population, the increasing incidence of diabetes and more complex percutaneous coronary interventions, resulting in operations being carried out on patients at higher risk. ${ }^{1}$ Clearly, new treatment strategies are required to protect the myocardium in these higher-risk patients undergoing CABG surgery, in order to improve clinical outcomes in this patient group.

In this regard, the endogenous cardioprotective strategy of remote ischaemic preconditioning (RIPC), a phenomenon in which brief ischaemia of one organ or tissue confers protection of another organ or tissue against a sustained ischaemiareperfusion injury insult, may be of benefit (reviewed by Hausenloy et $a l^{2}$ ). This phenomenon was initially described in 1993 by Przyklenk and colleagues, ${ }^{3}$ as intramyocardial protection extending from one coronary artery bed to another, but subsequent studies have demonstrated that brief ischaemia of distal organs and tissues such as the kidney, ${ }^{4}$ and small intestine ${ }^{5}$ reduce myocardial infarct size in animal hearts. Brief limb ischaemia was first used to induce RIPC in animals by Birnbaum and colleagues in $1997,{ }^{6}$ who reported cardioprotection using an invasive preconditioning stimulus comprising partial reduction in femoral artery flow applied in conjunction with pacing of the lower limb muscle. Kharbanda et al characterised a far less invasive transient upper limb ischaemia protocol in adult human volunteers and demonstrated an improvement in endothelial function in the contralateral upper limb. This technique was used in patients for the first time in children undergoing corrective surgery for congenital heart disease, in whom it was shown to reduce troponin release $24 \mathrm{~h}$ postoperatively. ${ }^{8} \mathrm{We}$ and others have recently demonstrated that RIPC using brief ischaemia and reperfusion of the arm or leg reduces myocardial injury in patients undergoing cardiac surgery ${ }^{9}$ or in surgical repair of an abdominal aortic aneurysm. ${ }^{10}$

For patients undergoing on-pump CABG surgery, cardioplegia or cross-clamp fibrillation are the standard techniques for protecting the heart during surgery, with the former approach in the form of cold-blood cardioplegia being predominantly used world wide. We have recently reported that RIPC reduces myocardial injury in patients undergoing elective CABG surgery in whom crossclamp fibrillation was the predominant method used to protect the heart during the surgical procedure. $^{9}$ 
Therefore, given that cardioplegia is the more commonly used method for myocardial protection during on-pump cardiac surgery ${ }^{11}{ }^{12}$ in this study we examined a new cohort of patients undergoing elective $\mathrm{CABG}$ receiving cold-blood cardioplegia alone in order to determine whether RIPC using brief limb ischaemia confers cardioprotection in a setting that is more clinically applicable.

\section{PATIENTS AND METHODS}

The study received local ethics committee approval and was carried out in accordance with the UCL Hospitals NHS Trust guidelines.

\section{Patient selection}

Adult patients with coronary artery disease consecutively referred for elective CABG surgery with or without concomitant aortic valve replacement between February 2007 and February 2008 were recruited. Patients over 80 years of age, with unstable angina, diabetes mellitus, hepatic, renal or pulmonary disease, were excluded, as were patients with peripheral vascular disease affecting the upper limbs. Written informed consent was obtained from all patients who entered into the study. Consenting patients were randomised to receive either control or RIPC before CABG surgery.

\section{The remote ischaemic preconditioning protocol}

RIPC comprised three 5 min cycles of right upper limb ischaemia, induced by a blood pressure cuff placed on the right upper arm and inflated to $200 \mathrm{~mm} \mathrm{Hg}$, with an intervening 5 min of reperfusion during which time the cuff was deflated. ${ }^{7}$ Control patients had a deflated cuff placed on the right upper arm for $30 \mathrm{~min}$. The RIPC protocol was applied after anaesthesia induction and before the start of surgery. Patients and the cardiac surgeons were blinded to treatment allocation, although the investigators and anaesthetists were not blinded.

\section{Surgical methodology}

Premedication comprising temazepam 10-20 mg orally was given to each patient $1 \mathrm{~h}$ before surgery. On arrival in the anaesthetic room, a peripheral venous cannula was inserted and patients were sedated with midazolam. An arterial cannula was inserted before induction of anaesthesia. Continuous arterial pressure monitoring was started and an infusion of Hartmann's solution was made. Anaesthesia was induced with midazolam with or without etomidate or propofol, fentanyl $(5-15 \mu \mathrm{g} / \mathrm{kg})$ and pancuronium $(0.1 \mathrm{mg} / \mathrm{kg})$. The trachea was intubated, mechanical ventilation started with $\mathrm{O}_{2}$ with or without air and anaesthesia was maintained either with halogenated anaesthetics or with a propofol infusion administered by target controlled infusion to achieve a target plasma concentration of 3-8 $\mu \mathrm{g} / \mathrm{ml}$. Midazolam, fentanyl and pancuronium were given as required. Arterial blood pressure, central venous pressure, leads I and III of the electrocardiogram and nasopharyngeal temperature were recorded continuously.

The surgical technique used was the same for both treatment groups (the surgeons were blinded to the treatment allocation). Non-pulsatile cardiopulmonary bypass was employed using a membrane oxygenator and cardiotomy suction in a standard manner. The CABGs (left internal mammary artery or saphenous vein grafts) were constructed on cardiopulmonary bypass with each anastomosis to the coronary arteries being performed with the aorta, using intermittent antegrade and/or retrograde cold-blood cardioplegia (one part of St Thomas cardioplegia solution mixed with four parts of blood). Coldblood cardioplegia was instilled into the aortic root after the aortic cross clamp was applied to the ascending aorta and repeated every $20 \mathrm{~min}$ until the distal coronary anastomoses were performed. After the aortic cross clamp was released, the proximal anastomoses were performed by applying a side-biting clamp. After construction of all the grafts, cardiopulmonary bypass was discontinued and protamine was used to reverse the effect of heparin.

\section{Serum troponin T measurement}

Blood samples for measurement of troponin $\mathrm{T}$ were taken preoperatively and 6, 12, 24, 48 and $72 \mathrm{~h}$ after surgery. Troponin $\mathrm{T}$ was measured quantitatively by a one-step enzyme immunoassay based on electrochemiluminescence technology (Elecsys 2010; Roche Diagnostics, UK). The lower detection limit of this assay is $0.01 \mu \mathrm{g} / \mathrm{l}$ with a recommended diagnostic range of 0.03 $0.09 \mu \mathrm{g} / 1$ indicating possible myocardial injury and a threshold of $\geqslant 0.1 \mu \mathrm{g} / 1$ indicating significant myocardial injury.

\section{Statistical analysis}

Data are presented as mean (SD). Comparison between treatment groups was made using the unpaired student test. A value of $p<0.05$ was considered significant. A sample size of at least 45 patients was determined based on the following assumptions: (a) that from our previous studies we would expect a difference in total serum troponin $T$ release over $72 \mathrm{~h}$ of about $15 \mu \mathrm{g} / \mathrm{l}$ between treated and untreated patients ${ }^{9} ;(b)$ a power of at least $80 \%$; (c) a standard deviation of $25 \mu \mathrm{g} / 1^{9}$ and (d) significance declared at the two-sided 5\% level. After consent had been obtained for participation in the clinical trial, patients were randomly assigned to receive either RIPC treatment or control. A computer-generated table of random numbers was used for randomisation. The analysis was by intention to treat. Standard statistical methods were used to test the differences between treatment groups-the independent $t$ test for continuous variables and the $\chi^{2}$ and Fisher exact test for categorical variables. The general linear regression model was used for multivariate analysis. Statistical analysis was performed using the SPSS statistical software, version 14.0

\section{RESULTS}

Sixty-one patients scheduled for elective CABG surgery were screened for eligibility, of whom 45 were recruited and randomised to intervention or control (fig 1). Of the 16 patients ineligible for inclusion in the study, 10 had diabetes mellitus and three had renal failure and were therefore excluded while three refused to participate. Forty-five patients who consented were recruited to the study and were randomised to receive either RIPC $(n=23)$ or control $(n=22)$ before CABG surgery. There was no difference in the baseline patient characteristics (table 1) between the two groups.

Five patients $(23 \%)$ in the control group had concomitant aortic valve replacement during CABG surgery as compared with only three patients (13\%) in the RIPC group (table 2). Aortic valve replacement was undertaken for severe aortic stenosis in all cases. The aortic cross-clamp time $(p=0.098)$ and bypass time $(p=0.218)$ were longer in the control group than in the RIPC group, although these differences were not statistically significant. The time taken from the termination of the RIPC protocol to the first aortic cross clamp was within $60 \mathrm{~min}$ for all patients. There were no untoward consequences of the RIPC protocol. 


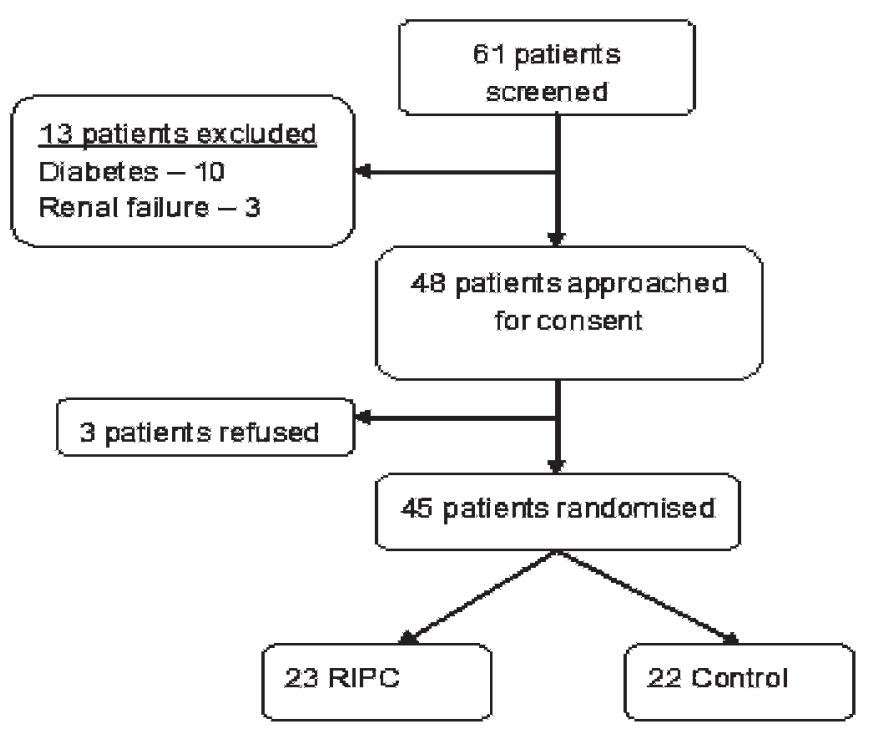

Figure 1 Clinical trial flow diagram. RIPC, remote ischaemic preconditioning.

Baseline preoperative troponin T levels were $<0.01 \mu \mathrm{g} / 1$ in both treatment groups. RIPC reduced the perioperative troponin $T$ release over the $72 \mathrm{~h}$ after cardiac surgery (fig 2). The total troponin $\mathrm{T}$ released, expressed as the area under the curve over the $72 \mathrm{~h}$ after surgery, was reduced from a mean (SD) of 31.53 (24.04) $\mu \mathrm{g} / 1.72 \mathrm{~h}$ in controls to 18.16 (6.67) $\mu \mathrm{g} / \mathrm{l} .72 \mathrm{~h}$ with RIPC (mean difference $13.37 \mu \mathrm{g} / 1.72 \mathrm{~h}, 95 \%$ CI 2.41 to $24.33 \mu \mathrm{g} / \mathrm{l}$, $\mathrm{p}=0.019)$ a reduction of $42.4 \%$. Importantly, this difference remained significant after correction for the differences in cardiac bypass time and cross-clamp time between the two groups using a general linear univariate regression model $(p=0.017)$. Of the above confounding variables, cardiac bypass time alone, which was longer in the control group, was an independent predictor of greater absolute troponin $\mathrm{T}$ release $(p=0.002)$.

\section{DISCUSSION}

In this clinical study, we demonstrate for the first time that RIPC induced by applying brief ischaemia and reperfusion to the forearm by a blood pressure cuff can reduce injury over and above the myocardial protection provided by the cold-blood cardioplegia. This was shown by the $42 \%$ reduction in the total serum troponin $\mathrm{T}$ release over the $72 \mathrm{~h}$ perioperative period. The magnitude of this cardioprotective effect is similar to that achieved in our recent clinical study in which we first reported cardioprotection with this RIPC protocol in a cohort of elective patients undergoing $C A B G$ receiving predominantly cross-clamp fibrillation as the method of myocardial preservation. ${ }^{9}$ It was essential to determine whether this non-invasive treatment strategy could confer cardioprotection over and above that offered by cold-blood cardioplegia, the technique most often used world wide for protecting the heart during on-pump cardiac surgery. In addition, since the results of conventional ischaemic preconditioning induced by aortic cross clamping are controversial in the setting of cardioplegia, ${ }^{13-18}$ it was important to establish the efficacy of RIPC in this clinical setting. An earlier study from our group compared the effect of invasive ischaemic preconditioning using short periods of aortic cross clamping in patients undergoing routine cross-clamp fibrillation and chemical cardioplegia during $\mathrm{CABG}$ and found that
Table 1 Patient characteristics

\begin{tabular}{|c|c|c|}
\hline Characteristics & Control (n= 22) & $\operatorname{RIPC}(n=23)$ \\
\hline \multicolumn{3}{|l|}{ Demographics } \\
\hline Age (years), mean (SD) & $64(9.0)$ & $62(9.7)$ \\
\hline Male & $19(86)$ & $19(83)$ \\
\hline Female & $3(14)$ & $4(17)$ \\
\hline Hypercholesterolaemia & $18(82)$ & $19(83)$ \\
\hline Hypertension & $13(59)$ & $19(83)$ \\
\hline Previous myocardial infarction & $5(23)$ & $5(22)$ \\
\hline Previous stroke & 0 & $1(4)$ \\
\hline Peripheral vascular disease & $1(5)$ & $1(4)$ \\
\hline \multicolumn{3}{|l|}{ Smoking history } \\
\hline Current smokers & $7(32)$ & $5(22)$ \\
\hline Ex-smokers & $8(36)$ & $10(43)$ \\
\hline Never smoked & $7(32)$ & $8(35)$ \\
\hline Family history of IHD & $11(50)$ & $7(30)$ \\
\hline Body mass index, mean (SD) & $28(3.9)$ & $26(3.5)$ \\
\hline NYHA class, mean (SD) & $1.4(0.8)$ & $1.7(0.8)$ \\
\hline CCS class, mean (SD) & $1.4(0.8)$ & $1.7(0.9)$ \\
\hline \multicolumn{3}{|l|}{ Ejection fraction } \\
\hline$>55 \%$ & $21(95)$ & $20(87)$ \\
\hline $35-55 \%$ & $1(5)$ & $2(9)$ \\
\hline$<35 \%$ & 0 & $1(4)$ \\
\hline Euroscore, mean (SD) & $2.6(2.1)$ & $2.1(1.9)$ \\
\hline \multicolumn{3}{|l|}{ Drug history } \\
\hline Aspirin & $12(55)$ & $10(43)$ \\
\hline$\beta$ Blocker & $16(73)$ & $17(74)$ \\
\hline Cholesterol-lowering drug & $18(82)$ & $19(83)$ \\
\hline $\begin{array}{l}\text { ACE-inhibitor/angiotensin receptor } \\
\text { blocker }\end{array}$ & $10(45)$ & $13(57)$ \\
\hline Nitrate & $3(14)$ & $3(13)$ \\
\hline
\end{tabular}

Values are presented as number (\%) unless stated otherwise.

CCS, Canadian Cardiovascular Society; IHD, ischaemic heart disease; NYHA, New York Heart Association; RIPC, remote ischaemic preconditioning.

conventional preconditioning was cardioprotective in both settings. ${ }^{19}$ Therefore both conventional and remote preconditioning appear to be beneficial in the setting of cardioplegia. However, conventional ischaemic preconditioning protocols involve intermittent aortic cross clamping before the insertion of the grafts and thus have not found favour with surgeons. Therefore this non-invasive technique of remote limb preconditioning which can be performed with minimum hindrance

Table 2 Details of cardiac bypass surgery

\begin{tabular}{lcc}
\hline Details & Control (n= 22) & RIPC (n= 23) \\
\hline Anaesthetic agent & & 17 \\
$\quad$ Isoflurane/sevoflurane & 17 & 6 \\
$\quad$ Propofol & 5 & $86(16)$ \\
Bypass time (min), mean (SD) & $97(38)$ & $53(14)$ \\
Total cross-clamp time (min), mean & $65(30)$ & \\
(SD) & & \\
Cardioplegia & 17 & 21 \\
$\quad$ Anterograde only & 5 & 4 \\
$\quad$ Anterograde+retrograde & $1(5)$ & $1(4)$ \\
Number of grafts: & $5(23)$ & $2(9)$ \\
$\quad$ One & $12(55)$ & $15(65)$ \\
$\quad$ Two & $4(18)$ & $5(22)$ \\
$\quad$ Three & $5(23)$ & $3(13)$ \\
$\quad$ Four &
\end{tabular}

Values are shown as number (\%) unless stated otherwise.

$\mathrm{RIPC}$, remote ischaemic preconditioning. 


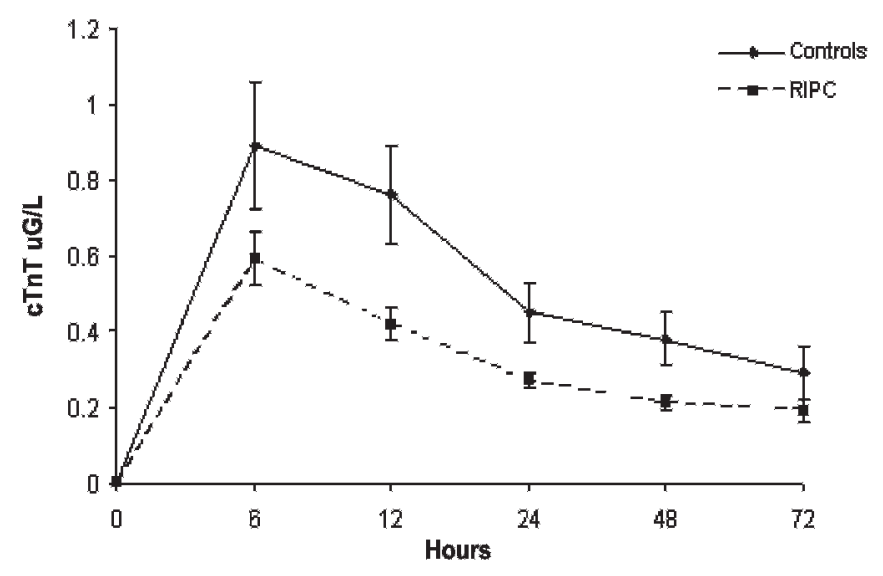

Figure 2 Remote ischaemic preconditioning (RIPC) reduced the area under the curve of troponin T over the $72 \mathrm{~h}$ perioperative period by $42 \%$ in adult patients undergoing elective coronary artery bypass graft surgery with cold-blood cardioplegia. Values presented are mean (SEM).

${ }^{*} p<0.05$ compared with control. RIPC $(n=23)$ and controls $(n=22)$. cTnT, cardiac troponin T.

before the patient enters the operation theatre may be more widely acceptable.

Myocardial injury as indicated by the perioperative release of cardiac enzymes during cardiac surgery occurs as a result of combined ischaemia-reperfusion injury encountered during aortic cross clamping, coronary microembolisation ${ }^{20}$ and direct myocardial damage from handling of the heart. Cardiac-specific markers like troponin T, troponin I and CK-MB have been used to quantify this form of myocardial injury and have been reported by several clinical studies to be associated with worse short-term and long-term outcomes. ${ }^{21-23}$ Lehrke and colleagues ${ }^{21}$ reported in a case series of 204 patients undergoing elective CABG that perioperative troponin $T$ release was associated with worse clinical outcomes, such that a $48 \mathrm{~h}$ post-surgery serum troponin $\mathrm{T}$ level of $\geqslant 0.46 \mu \mathrm{g} / \mathrm{l}$ was associated with the greatest risk as shown by a 4.9-fold higher long-term risk for subsequent cardiac death. Indeed, the recent new universal definition of myocardial infarction has recognised the importance of postoperative enzyme release and defined this as type 5 myocardial infarction associated with poor clinical outcomes. ${ }^{24}$ We have demonstrated a reduction in myocardial injury in low-risk patients undergoing cardiac surgery. High-risk patients tend to have greater myocardial injury ${ }^{25}$ and therefore may derive greater benefit from RIPC, but this remains to be determined.

RIPC has been shown to have protective effects in several tissues in a number of animal studies including the liver, gut, pancreas, brain, lung (reviewed by Hausenloy et $a l^{2}$ ). Therefore, it appears that applying brief ischaemia and reperfusion in one organ or tissue (in this case the upper limb) confers systemic protection in multiple organ systems against the detrimental effects of acute ischaemia-reperfusion injury. Ali et $a l^{10}$ have recently demonstrated that intermittent lower limb ischaemia by cross clamping the iliac artery reduces both the cardiac and renal injury sustained in patients undergoing abdominal aortic aneurysm repair. Thus, in the setting of cardiac surgery, RIPC has the potential to reduce not only myocardial injury, but also renal, cerebral and pulmonary side effects, which in turn can improve short- and long-term outcomes.

The mechanisms underlying RIPC are yet to be elucidated, although animal studies have thus far implicated either a humoral or neuronal pathway as the mediator of protection. On the one hand, the abrogation of remotely induced cardioprotection with the ganglion blocker hexamethonium ${ }^{26}$ or the pretreatment of sensory nerves with capsaicin, ${ }^{27}$ suggested a neuronal mechanism. On the other hand, Konstantinov et a ${ }^{28}$ demonstrated that RIPC can also protect the denervated transplanted heart, which would suggest a humoral mechanism. Several humoral mediators have indeed been implicated, including opioids, calcitonin gene-related peptide, insulin, bradykinin, adenosine and endocannabinoids (reviewed by Hausenloy et $a l^{2}$ ). These humoral mediators may be released by the remote ischaemic tissue and be carried to the target tissue in the blood stream or be released locally in the target tissue, making them available to activate neural pathways. An interesting recent experimental study has implicated prosurvival kinase signalling within the remote preconditioning organ as a mediator of cardioprotection. Heidbreder et al ${ }^{29}$ reported that brief mesenteric artery occlusion reduced myocardial infarct size and that this cardioprotective effect was dependent on the phosphorylation of p38MAPK, Erk1/2 and JNK within the small intestinal tissue.

In summary, this single-blind randomised controlled clinical study was designed to determine whether RIPC using brief ischaemia of the forearm can reduce myocardial injury in elective patients undergoing CABG receiving cold-blood cardioplegia during surgery, making the positive findings more widely applicable, given that cardioplegia is the more commonly used method for myocardial preservation during cardiac surgery. We confirm that RIPC can reduce myocardial injury over and above that provided by cold-blood cardioplegia as indicated by a $42 \%$ reduction in troponin $T$ released over the $72 \mathrm{~h}$ perioperative period. Further studies are underway to investigate whether this cardioprotective strategy is beneficial in patients undergoing off-pump CABG surgery. Large multicentre clinical studies are clearly required in order to determine whether the reduction in myocardial injury observed in recent clinical studies investigating this form of RIPC impacts on clinical outcomes in patients undergoing cardiac surgery.

Acknowledgements: We thank the British Heart Foundation for their continued support, and would like to thank the patients and staff at the Heart Hospital for their assistance throughout this study.

Funding: This work was undertaken at UCLH/UCL who received a proportion of funding from the Department of Health's NIHR Biomedical Research Centres funding scheme.

Competing interests: None.

Ethics approval: Local ethics committee approval received.

Provenance and peer review: Not commissioned; externally peer reviewed.

\section{REFERENCES}

1. Biancari F, Kangasniemi OP, Mahar MA, et al. Changing risk of patients undergoing coronary artery bypass surgery. Interact Cardiovasc Thorac Surg 2008 May 21. [Epub ahead of print]

2. Hausenloy DJ, Yellon DM. Remote ischaemic preconditioning: underlying mechanisms and clinical application. Cardiovasc Res 2008;79:377-86.

3. Przyklenk K, Bauer B, Ovize M, et al. Regional ischemic 'preconditioning' protects remote virgin myocardium from subsequent sustained coronary occlusion. Circulation 1993;87:893-9.

4. Pell TJ, Baxter GF, Yellon DM, et al. Renal ischemia preconditions myocardium: role of adenosine receptors and ATP-sensitive potassium channels. Am J Physiol 1998;44:H1542-7.

5. Gho BC, Schoemaker RG, van den Doel MA, et al. Myocardial protection by brief ischemia in noncardiac tissue. Circulation 1996;94:2193-200.

6. Birnbaum Y, Hale SL, Kloner RA. Ischemic preconditioning at a distance: reduction of myocardial infarct size by partial reduction of blood supply combined with rapid stimulation of the gastrocnemius muscle in the rabbit. Circulation 1997;96:1641-6.

7. Kharbanda RK, Mortensen UM, White PA, et al. Transient limb ischemia induces remote ischemic preconditioning in vivo. Circulation 2002;106:2881-3. 
8. Cheung MM, Kharbanda RK, Konstantinov IE, et al. Randomized controlled trial of the effects of remote ischemic preconditioning on children undergoing cardiac surgery: first clinical application in humans. J Am Coll Cardiol 2006:47:2277-82.

9. Hausenloy DJ, Mwamure PK, Venugopal V, et al. Effect of remote ischaemic preconditioning on myocardial injury in patients undergoing coronary artery bypass graft surgery: a randomised controlled trial. Lancet 2007:370:575-9.

10. Ali ZA, Callaghan CJ, Lim E, et al. Remote ischemic preconditioning reduces myocardial and renal injury after elective abdominal aortic aneurysm repair: a randomized controlled trial. Circulation 2007;116:198-105.

11. Karthik S, Grayson $A D, 0$ o $A Y$, et al. A survey of current myocardial protection practices during coronary artery bypass grafting. Ann R Coll Surg Engl 2004;86:413-5.

12. Palmer G, Herbert MA, Prince SL, et al. Coronary Artery Revascularization (CARE) registry: an observational study of on-pump and off-pump coronary artery revascularization. Ann Thorac Surg 2007:83:986-91.

13. Perrault LP, Menasche P, Bel A, et al. Ischemic preconditioning in cardiac surgery: a word of caution. J Thorac Cardiovasc Surg 1996;112:1378-86.

14. Kaukoranta PK, Lepojarvi MV, Kiviluoma KT, et al. Myocardial protection during antegrade versus retrograde cardioplegia. Ann Thorac Surg 1998;66:755-61.

15. Cremer J, Steinhoff G, Karck M, et al. Ischemic preconditioning prior to myocardial protection with cold blood cardioplegia in coronary surgery. Eur J Cardiothorac Surg 1997; 12:753-8.

16. Illes RW, Swoyer KD. Prospective, randomized clinical study of ischemic preconditioning as an adjunct to intermittent cold blood cardioplegia. Ann Thorac Surg 1998;65:748-52.

17. Lu EX, Chen SX, Yuan MD, et al. Preconditioning improves myocardial preservation in patients undergoing open heart operations. Ann Thorac Surg 1997;64:1320-4.

18. Wu ZK, Tarkka MR, Pehkonen E, et al. Ischaemic preconditioning has a beneficial effect on left ventricular haemodynamic function after a coronary artery biopass grafting operation. Scand Cardiovasc J 2000;34:247-53.
19. Teoh LK, Grant R, Hulf JA, et al. A comparison between ischemic preconditioning, intermittent cross-clamp fibrillation and cold crystalloid cardioplegia for myocardial protection during coronary artery bypass graft surgery. Cardiovasc Surg 2002;10:251-5

20. Heusch G, Schulz R, Haude M, et al. Coronary microembolization. J Mol Cell Cardiol 2004;37:23-31.

21. Lehrke S, Steen H, Sievers HH, et al. Cardiac troponin T for prediction of short- and long-term morbidity and mortality after elective open heart surgery. Clin Chem 2004;50:1560-7.

22. Fellahi JL, Gue X, Richomme $X$, et al. Short- and long-term prognostic value of postoperative cardiac troponin I concentration in patients undergoing coronary artery bypass grafting. Anesthesiology 2003;99:270-4.

23. Brener SJ, Lytle BW, Schneider JP, et al. Association between CK-MB elevation after percutaneous or surgical revascularization and three-year mortality. J Am Coll Cardiol 2002; $40: 1961-7$

24. Thygesen K, Alpert JS, White HD, et al. Universal definition of myocardial infarction. Circulation 2007:116:2634-53

25. Kathiresan S, Servoss SJ, Newell JB, et al. Cardiac troponin T elevation after coronary artery bypass grafting is associated with increased one-year mortality. Am J Cardiol 2004;94:879-81.

26. Gho BC, Schoemaker RG, van den Doel MA, et al. Myocardial protection by brief ischemia in noncardiac tissue. Circulation 1996:94:2193-200.

27. Tang ZL, Dai W, Li YJ, et al. Involvement of capsaicin-sensitive sensory nerves in early and delayed cardioprotection induced by a brief ischaemia of the small intestine. Naunyn Schmiedebergs Arch Pharmacol 1999;359:243-7.

28. Konstantinov IE, Li J, Cheung MM, et al. Remote ischemic preconditioning of the recipient reduces myocardial ischemia-reperfusion injury of the denervated donor heart via a Katp channel-dependent mechanism. Transplantation 2005;79:1691-5.

29. Heidbreder M, Naumann A, Tempel K, et al. Remote vs. ischaemic preconditioning the differential role of mitogen-activated protein kinase pathways. Cardiovasc Res 2008;78:108-15. 